\title{
Die Psychologie des Lügens
}

La psychologie du mensonge

The psychology of lying

Jochen Jordan

\section{CpenEdition}

\section{Journals}

Édition électronique

URL : http://journals.openedition.org/ceg/1656

DOI : $10.4000 /$ ceg. 1656

ISSN : 2605-8359

\section{Éditeur}

Presses Universitaires de Provence

Édition imprimée

Date de publication : 1 décembre 2014

Pagination : 45-61

ISSN : 0751-4239

\section{Référence électronique}

Jochen Jordan, «Die Psychologie des Lügens », Cahiers d'Études Germaniques [Online], 67 | 2014,

Online erschienen am: 17 Dezember 2017, abgerufen am 10 Dezember 2020. URL : http://

journals.openedition.org/ceg/1656; DOI : https://doi.org/10.4000/ceg.1656 


\title{
Die Psychologie des Lügens
}

\author{
Jochen JORDAN \\ Kerckhoff Klinik Bad Nauheim und Universitätsklinik Frankfurt am Main
}

Die Lüge ist die Visitenkarte des Menschen. Männer lügen häufiger als Frauen, Frauen lügen anders als Männer, in verschiedenen Kulturen und Religionen gibt es unterschiedliche Motive und Lügenkonstruktionen und über die Jahrhunderte ändern sich die Gepflogenheiten rund ums Lügen, wobei weltweit auch heute noch alle denkbaren Umgangsweisen vorkommen: in einigen Teilen der Welt werden Frauen gesteinigt und ermordet, wenn sie in bestimmten Fragen lügen, in anderen Ländern würde man sich wundern, wenn Menschen in spezifischen Kontexten aufrichtig sind. Weltweit ist die Lüge die „Corporate Identity“ spezifischer Berufsausübungen, insbesondere in Politik, Banken, Religionsgemeinschaften und Werbeindustrie. Als Bestandteil des Alltagsrepertoires der Kommunikation versteckt sie sich in jeder charmanten Schmeichelei oder tritt niederträchtig und zerstörerisch in Erscheinung. Das folgende Kapitel beschäftigt sich mit dem Motivsträngen, den Konstruktionen und Gefahren des Lügens ebenso wie mit den Versuchen, die Lüge sicher zu erkennen und Lügner zu überführen.

In jedem Land Europas finden öffentliche Auseinandersetzungen um Wahrheit und Lüge statt, die zuweilen bizarre sprachliche Gebilde hervorbringen. Der deutsche Kurzzeitbundespräsident Wulff kreierte beispielsweise folgende Formulierung: „Ich habe in diesem Zusammenhang nicht gelogen, sondern nur die halbe Wahrheit gesagt.“

$\mathrm{Ob}$ im Kontext von Nixons Watergate-Affäre, Strauss-Kahns ganz anderer Affäre in New York, von Boris Beckers Erlebnis in einer Wäschekammer oder Clintons Petting im Oval-Office, alles sind Lehrstücke der allgemeinen Grammatik der Lüge.

\section{Literaturrecherche}

Literaturrecherchen in den großen wissenschaftlichen Datenbanken führen zu unüberschaubar vielen psychologischen Originalarbeiten zum Thema. Der vorliegende Beitrag ist keine systematische oder vollständige 
Literaturdarstellung, sondern eine subjektive Auswahl einiger interessanter Aspekte.

\section{Eine psychologische Definition der Lüge}

Die Definition enthält drei Merkmale, ohne die eine nicht handhabbare Uferlosigkeit des Themas entstünde. Eine Definition von „Wahrheit“ wird hier nicht versucht, obgleich die Begriffe Lüge und Wahrheit eine untrennbare Einheit bilden. Der Wahrheitsbegriff wird der Philosophie überlassen. ${ }^{1}$ Mit der Festlegung auf drei Definitionsmerkmale wird ein erheblicher Teil der psychologischen Literatur bewusst ausgeklammert, weil andernfalls der Begriff nicht mehr operationalisierbar wäre.

Die Lüge ist ein Verhalten mit folgenden Charakteristika:

1. Es werden bewusst und wissentlich unwahre Zusammenhänge postuliert. Die Bedingung des bewussten Entschlusses bedeutet, dass der Handelnde immer die Möglichkeit der Entscheidung zwischen Wahrheit und Lüge hat.

2. Es gibt für Sender und Empfänger eine relevante, eindeutig klärbare Differenz zwischen (objektiv) nachvollziehbaren Sachverhalten und deren Darstellung durch den Lügner.

3. Es ist entscheidend, dass der Empfänger der Lüge (das Opfer) vorher keine Ankündigung des Lügners erhalten und auch kein klares Einverständnis gegeben hat. $^{2}$

Zur Verdeutlichung sind einige Abgrenzungen hilfreich:

a. Ein Mensch mit einer halluzinatorischen Psychose lügt demnach nicht, wenn er Dinge ausspricht, die der Wahrheit nicht entsprechen.

b. Ein pathologischer Hochstapler muss als Lügner angesehen werden. Er unterliegt zwar einem pathologischen, oft nicht bzw. sehr schwer kontrollierbaren Drang, seine Selbstdarstellung forciert vorzutragen und auszugestalten. Er weiß im Detail dennoch, dass seine Geschichten erfunden und massiv ausgestaltet sind. Er ist sich also der Lüge bewusst und daher straffähig, denn sein Handeln ist bewusstseinsfähig und prinzipiell beeinflussbar (z.B. durch erlernbare Distanzierungstechniken, regelmäßige Psychotherapie und/oder Medikamente).

c. Genauso wenig ist der Zauberer ein notorischer Lügner, wenn er sein Publikum ein- ums andere Mal hinters Licht führt, so wenig wie ein

\footnotetext{
${ }^{1}$ Konrad Paul LiESMANN (Hrsg.), Der Wille zum Schein. Über Wahrheit und Lüge, Reihe: Philosophicum Lech, Bd. 8, Wien, Zsolnay, 2005. Arno BARUZZI, Philosophie der Lüge, Darmstadt, Wissenschaftliche Buchgesellschaft, 1996.

${ }^{2}$ Dieser Aspekt der Positionierung des Opfers ist wesentlich, weil es eine Vielzahl von „betrügerischen“ Konstellationen gibt, die durch das Einverständnis des Opfers charakterisiert sind und legitimiert werden.
} 
Schauspieler wenn er Hass und Trauer nur vorspielt, ohne sie zu empfinden. Das Liebeswerben wie auch so manche Liebeserklärung sind mindestens Teillügen. Sie werden dennoch nicht als solche markiert, weil sie beim „Opfer“ Glückshormone ausschütten.

\section{Der Beitrag der Psychoanalyse}

Der Platzmangel im vorliegenden Kontext erlaubt nur einige kurze Anmerkungen. Diejenigen Stellen, an denen sich Freud mit der Lüge beschäftigt, haben eine spezifische Argumentationslogik: Sie sind dadurch charakterisiert, dass sie v.a. den unbewussten Anteil des Lügens herausarbeiten und hier besonders die Bedeutung sexueller Impulse. Eindrucksvolle, wenn auch kurze Anmerkungen zur Lüge finden sich an wenigen Stellen. $\mathrm{Zu}$ nennen ist ein von Freud wiedergegebener Judenwitz (der wirklich schwer zu verstehen ist). ${ }^{3}$ Weiterhin finden sich Anmerkungen in der Arbeit Die Zukunft einer Illusion, in der er sich mit den kulturellen Normen und Verboten und deren regelmäßiger Außerachtlassung beschäftigt. ${ }^{4}$ In zwei Fallbeispielen von lügenden Kindern ${ }^{5}$ wird die Frage erörtert, warum Kinder chronisch und teilweise selbstschädigend lügen und welche unbewussten Entwicklungskonflikte dahinter verborgen sind. ${ }^{6}$ Freud geht so weit, dass er formuliert, dass auch das Unbewusste selbst lügen kann (womit dann aber eine bewusste Entscheidung ausgeschlossen ist, die der Kern der hier vorgetragenen Definition ist).

\section{Die Allgemeine Psychologie der Lüge}

Da Lügen ein zentrales Verhaltensmerkmal zwischenmenschlicher Beziehungsgestaltung ist, gehört das Handwerkszeug des Lügens zur Grundausstattung und muss schon in frühester Kindheit trainiert werden. ${ }^{7}$ Das psychologische Paradoxon ist: Obgleich die Lüge alltäglich ist, so wird sie doch in allen Kulturen als moralisch verwerflich klassifiziert. Dies impliziert

\footnotetext{
${ }^{3}$ Sigmund Freud, Der Witz und seine Beziehung zum Unbewussten, Studienausgabe IV, Frankfurt/M., Fischer, 1982, S. 109.

${ }^{4}$ Sigmund Freud, Die Zukunft einer Illusion, Studienausgabe IX, Frankfurt/M., Fischer, 1974, S. 145f.

Sigmund Freud, Zwei Kinderlügen, Studienausgabe V, Frankfurt/M., Fischer, 1975, S. 231-234.

${ }^{6}$ In der wissenschaftlichen Literatur zur Entwicklungspsychologie oder pädagogischen Psychologie wird dies häufig in ähnlicher Weise erörtert, vgl. Maurice KROUT, „The psychology of childrens's lies“", in The Journal of Abnormal and Social Psychology, 26, 1, 1.27, 1931.

${ }^{7}$ Betrachtet man die Gruppendynamik unserer Vorfahren (Bonoboaffen und Schimpansen) so sind hier deutliche Vorformen der Lüge zu finden und es ist mit hoher Wahrscheinlichkeit davon auszugehen, dass die Lüge auch phylogenetisch früh kultiviert wurde.
} 
unausweichliche intrapsychische Konflikte. Es ist zu vermuten, dass die negative Bewertung zivilisatorisch den Zweck hat, das Lügen ein wenig einzudämmen.

Interessant ist wissenschaftsgeschichtlich, dass in den entsprechenden Lehrbüchern der Psychologie regelmäßig ein entsprechendes Kapitel fehlt. In der Subdisziplin der „Allgemeinen Psychologie“, in welcher Sprache, Denken, Lernen und Verhalten beheimatet sind, wäre bei der Alltäglichkeit des Phänomens ein Kapitel zur Lüge zu erwarten. Und ein weiteres bemerkenswertes Charakteristikum psychologischer Forschung zur Lüge verdient der Markierung: Das Hauptinteresse gilt nicht der Entstehung und intrapsychischen Dynamik der Lüge, ${ }^{8}$ sondern deren Erkennen und Aufdecken (Körpersprache, Lügendetektor, etc. s.u.).

Die oben beschriebene Doppelmoral zeigt sich zunächst eindrucksvoll darin, dass die psychologische Forschung nahe legt, dass diejenigen Menschen, die von anderen als ehrlich, authentisch und offen - also wenig lügend - eingeschätzt werden, besonderes soziales Ansehen genießen. Nichtlügen gehört zu den sozial am meisten geschätzten Eigenschaften.

Lügen ist im Kern (sozusagen losgelöst vom konkreten Kontext) die Manipulation des anderen Menschen (ohne dass dieser es bemerkt also) mit dem Zweck, einen Vorteil daraus zu erreichen. Und (ganz wesentlich): Der erzielte Vorteil soll nicht vollständig gezahlt werden. Der Aufwand des Lügens wird also geringer eingeschätzt als die Mühe der Offenheit. Lüge und Täuschung erscheinen dem Lügenden als eine „preiswerte“ Vorteilsnahme.

Da Lügen trotz seiner Alltäglichkeit als moralisch verwerflich angesehen wird und da die Aufdeckung einer Lüge mit viel Ungemach verbunden ist, kann geschlossen werden, dass jede Lüge als intrapsychischer Vorgang mit einem energetisch aufwändigen, Zeit und Aufmerksamkeit kostenden Prozess verbunden ist. Lügen ist Stress und Stress löst eine Vielzahl körperlicher Reaktionen aus. Und zugleich werden - bei halbwegs gelungener Sozialisation - immer innere Konflikte und ein individuell unterschiedliches Quantum an Schuldgefühlen damit erkauft. Der je individuelle Preis der Lüge errechnet sich also aus der je spezifischen Persönlichkeitsstruktur.

Es ist festzuhalten: Lügen kostet das Gehirn Energie und Zeit (es blockiert also andere Prozesse im Gehirn) und löst zahlreiche körperliche Reaktionen aus. Es bedarf eines komplizierten Vorgangs der Güterabwägung und - wenn man nicht eine allzu schnelle oder leichte Überführung riskieren möchte bedarf es einer erheblichen sozialen Intelligenz: Man muss die Fähigkeit besitzen, den sozialen Kontext differenziert zu analysieren, man muss zur Perspektivenübernahme fähig sein, um vorauszuberechnen, wie der Empfänger der Lüge die Situation erlebt und bewertet. Weiterhin ist eine beachtliche Merkfähigkeit erforderlich, weil Nachfragen vom Opfer oder von Dritten zu erwarten sind und Widersprüche tunlichst vermieden werden

\footnotetext{
8 Leonard SAXE, „Lying. Thoughts of an applied social psychologist“, American Psychologist, 46, 4, S. 409-415, 1991.
} 
müssen, da sie immer der Anfang der Aufdeckung sind. Zusätzlich, und das ist der schwierigste Teil des Prozesses, muss ein hoher Aufwand betrieben werden, damit man sich nicht beim Vorgang des Lügens durch unpassende Tonlage, durch Sprechpausen oder inadäquate Mimik, Gestik und Körperhaltung verrät.

Der skizzierte intrapsychische Konflikt bildet natürlich soziale Normen ab und ist auf komplexe, aber widersprüchliche Weise gesellschaftlich kontextualisiert. Kinder lernen in der Schule, dass Aufrichtigkeit erwartet wird und Zuwiderhandlung nachteilig ist, während zugleich jede Nachrichtensendung schwerste folgenlose Lügen dokumentiert. Eine gesellschaftlich hoch angesehene Institution zelebriert seit Jahrhunderten die Doppelmoral: die Institution nämlich, die moralisch die Lüge geißelt und in der Sozialisation einen beträchtlichen Aufwand treibt, um die Lüge als verwerflich zu brandmarken, ist zugleich diejenige, die sich von Anbeginn an virtuos der Lüge bedient. Die katholische Kirche verwendet diese Sozialstrategie (Doppelmoral) routiniert seit Jahrtausenden. Beispiel: Sie betrieb in Deutschland lange Jahre Beratungsstellen für ungewollt Schwangere und bescheinigte diesen ihren Besuch, sagte aber offiziell, dieser Schein sei kein Schein im eigentlichen Sinne des Gesetzes, obgleich er vom Staat als solcher anerkannt wurde: es war der Schein-Schein erfunden („Ceci n'est pas une pipe“). Und noch raffinierter: Sie geißelt die Lüge und stellt zugleich den Lügenden ein Ritual zur Verfügung, das den inneren Konflikt minimiert: die Beichte. So kann man vor den Augen Gottes lügen und erhält durch Buße ein leichteres Gewissen, also Über-Ich-Entlastung.

In diesem Kontext muss eindringlich auf die enorm destruktive Kraft des Lügens hingewiesen werden. So alltäglich die Lüge sein mag, sie kann in bestimmten Kontexten eine dramatisch zerstörerische Seite haben: Bestimmte Lügen in spezifischen Kontexten können Menschen zerstören, in den Selbstmord treiben und für den Rest des Lebens ein Vertrauen in das eigene Ich, in die eigene Wahrnehmung und die Antizipierbarkeit des Lebens nehmen. Lügen können also andere Menschen zerstören und der Lügende hat meist die Fähigkeit, dies zu antizipieren. Lügen können eine massive Aggression sein und nicht reparierbare Schäden verursachen und zwar im persönlichen wie auch im gesellschaftlichen Lebensraum.

\section{Motivstränge des Lügens}

Die Komplexität und Vielfältigkeit der motivationalen Hintergründe des Lügens führen dazu, dass in der Literatur verschiedene Klassifikationsversuche unternommen wurden. Häufig werden die Motivstränge in drei Kategorien eingeteilt: Senderbezogene, empfängerbezogene und beziehungsbezogene Motive. Diese nachvollziehbare Einteilung hat den Nachteil erheblicher Überschneidungen. So können senderbezogene Motivstränge natürlich nie unabhängig von den sozialen Einbindungen 
verstanden werden, sie sind also immer auch empfänger- und beziehungsbezogen.

Nachfolgend wird folgende Systematisierung vorgenommen: zunächst werden das Lügenmotiv der Machterlangung und -erhaltung, dann die aus der innerpsychischen Balance resultierenden Motivstränge und schließlich die überwiegend beziehungsregulierenden Lügen dargestellt.

\section{Das Motiv der Machterlangung und -erhaltung}

Aus Sicht der Menschheitsgeschichte ist dies sicher die älteste Motivstruktur. Wenngleich die Frage der Bewusstheit nicht beantwortbar ist, kann die Verhaltensforschung zeigen, dass in der Regulation der Gruppenstruktur der Schimpansen bereits geschickt eingefädelte Täuschungsmanöver vorkommen.

Gesellschaftliche Macht, eine hierarchische Position, finanzielle Vorteile und Konkurrenzstreben sind sicherlich die häufigsten und gängigsten Motive für Täuschung und Lüge. Hier gibt es kaum Schranken und Hemmungen. Selbst die Aufdeckung von Lug und Betrug ist nur in seltenen Fällen wirklich für den Sender nachteilig (oft reicht es, wenn man einige Monate abtaucht oder eine erhebliche Geldstrafe akzeptiert; vgl. Franz Josef Strauß oder Guttenberg in Deutschland). Die Politik hat eigene Gesetze: Zuweilen lässt es sich mit offenkundigen Lügen lange und gut leben bzw. regieren. Allerdings führen hin und wieder spezifische Interessenlagen dazu, dass die Aufdeckung von im Grunde bekannten Lügen plötzlich zum Skandal oder zum Rücktritt führen.

Andererseits: Unsere Gesellschaft ist geprägt von zentralen ideologischen Lügenkonstruktionen, die fundamental und konstituierend unser politisches System bestimmen und die hierdurch natürlich individuelle Niederschläge haben. Hierzu gehören Lügen, wie etwa das Wort von der ,sozialen Marktwirtschaft" oder den ,gleichen Bildungschancen“.

So wie die Macht-Lüge im gesellschaftlichen Machtkontext ein konstitutives Element ist, so ist sie auch im zwischenmenschlichen Bereich eine der verbreitetsten Verhaltensstrategien: Vom Kindergarten bis ins Seniorenheim kommt die Lüge bei der Regulierung von sozialen Hierarchien und hinsichtlich des Zugangs zu relevanten Vorteilen zum Einsatz: Verunglimpfung, realer und geistiger Diebstahl, Aktenmanipulation und Mobbing sind einige Beispiele.

Wir versuchen Kindern und Jugendlichen mühsam zu vermitteln, dass es nicht in Ordnung ist, wenn man einem anderen Menschen gleich die Zähne ausschlägt oder ihm ein Stuhlbein über den Schädel zieht, wenn man etwas durchsetzen will. Wir erziehen zur Affektkontrolle, zum sozialen Dialog und zum Diskurs im Sinne von Habermas, während gleichzeitig Präsidenten von Staaten sich über Lügengeschichten den Zugang zu anderen Territorien verschaffen und ganze Völker und Kontinente mit Krieg überziehen. Fast 
immer stellt sich heraus, dass die veröffentlichten Begründungen auf bewusstem Betrug beruhten, was im Allgemeinen folgenlos bleibt.

\section{Die Lüge im Dienste der narzisstischen Gleichgewichtsregulation}

Da das Nicht-Lügen ein hohes Gut ist, gilt es als normatives Ideal. Intrapsychisch bedeutet dies, dass der Lügner eine Abwägung vornehmen muss, was seine Selbstbildbalance angeht: Der ständige Abgleich zwischen Real-Ich, Ideal-Ich und Wunsch-Ich (nämlich: wie möchte ich, dass andere mich sehen) erfordert nicht nur Zeit und Energie, sondern auch schwierige (weil konflikthafte) Entscheidungen, also im weitesten Sinne eine Güterabwägung. Die von Foucault beschriebene zivilisatorische Verinnerlichung psychischer Steuerungsmechanismen von (vormals durch äußere Kontrolle und Zwang herbeigeführten) Verhaltensmustern ist heute zunehmend wichtig. Anders ausgedrückt: Die Entscheidung, ob man im konkreten Fall lügen sollte, basiert nicht allein auf der Frage, wie sie sich sozial auswirken würde (also wie andere über mich urteilten, würde sie aufgedeckt), sondern auch auf der Frage, wie das innere Gleichgewicht betroffen sein würde (wie also mein Bild von mir aussähe).

Die je spezifische innere Balance (bzw. Dysbalance) eines Menschen ist demnach ein wesentlicher Motor (oder Bremser) der Täuschung und Lüge. Es ist hier vom Narzissmus nicht als einer klinischen Symptomatik die Rede, sondern vom Narzissmus als persönlichkeitsspezifische Eigenschaft der Balance zwischen Selbstvertrauen und Selbstzufriedenheit auf der einen und Mangel an Selbstbewusstsein und Minderwertigkeitsgefühlen auf der anderen Seite. An beiden Polen sind die Motive und Ausgestaltungen von Täuschung und Lüge deutlich anders.

Dient die Lüge dem narzisstischen Größenwahn, so ist sie dadurch motiviert, dass der Lügende sich damit noch weiter erhöhen möchte, sich über Andere stellen und weitere Glanzpunkte erlangen will. Nicht selten spielt dabei auch eine Rolle, dass nicht nur etwas erreicht wird, sondern dass man sich auch großartig fühlt, weil man in der Lage ist, andere Menschen erfolgreich zu manipulieren, ohne dass diese es merken. Es ist demnach ein narzisstischer Sieg über die ahnungslosen belogenen Personen, der der eigenen Regulation dient. Die Bewunderung, die glänzenden Augen des Gegenübers und der kurze Moment der Überlegenheit motivieren entsprechende Handlungen (da Glückshormone ausgeschüttet werden, kann man auch von einem nicht-Substanz-gebundene Suchtverhalten sprechen). Hier gibt es harmlose und durchschaubare Inszenierungen des Alltags, aber auch große katastrophale und desaströse Inszenierungen, die erheblichen Schaden anrichten und durchaus ruinös sein können. An beiden Enden der narzisstischen Dimension ist die psychische Stabilität des Lügenden nicht besonders groß und daher ist die Gefahr der Aufdeckung groß. Narzissten beider Ausprägungen lügen im Allgemeinen schlecht, weil sie - wie das Wort 
sagt - zu sehr auf sich bezogen sind und daher die Perspektivenübernahme mangelhaft ist. So erleiden sie oft die Scham der Aufdeckung und ihr Dilemma wird vergrößert und der Gewinn der Lüge ist spärlich und allzu kurzfristig (was wiederum, sozusagen kompensatorisch, die nächste „narzisstische Show“ auslöst).

Ein Beispiel für einen intrapsychischen Konflikt auf der Ebene des labilen Selbstbildes am anderen Pol der narzisstischen Regulation soll folgende Konstellation verdeutlichen: Ein Mensch mit einem rigiden strengen und unnachgiebigen Über-Ich hat dieses erfahrungsgemäß durch konkrete Umstände und Menschen gebildet. ${ }^{9}$ Man würde annehmen, dass er das Lügen in jedem Fall vermeidet (muss), um den zwangsläufigen inneren SelbstbildKonflikt zu vermeiden. So weit wäre der Fall einfach und klar: Unser guter Mensch führt ein weitgehend untadeliges Leben.

Aber unsere Psyche ist voller Ambivalenz, Konflikte sind selten zu befrieden, sie kehren wieder und inszenieren sich unbewusst: Unser braver Mensch hat mit hoher Wahrscheinlichkeit einen schweren inneren Autonomiekonflikt. Von den Eltern muss man sich eines Tages lösen. Je strenger das Über-Ich, desto problematischer gestaltet sich dies. Und so mancher psychischer Apparat gerät hier ins Straucheln und die Lüge wird scheinbar vielversprechend zum Instrument der Gewinnung von mehr innerem Raum, die Lüge verspricht also zuweilen Freiheit und Autonomie: Der innere Dialog geht ungefähr so: „Wenn man mich in eine solche Lage bringt (ich bin Opfer), dann ist das entwürdigend und wenn ich lüge, erhalte ich meinen Stolz sowie meine Selbstachtung und ich unterwerfe mich daher nicht".

Gemein wie das Leben so ist, geht alles schief und endet schlimmer als es am Anfang war: Die Lüge wird aufgedeckt, die Scham und Schande ist besonders groß und die erhoffte Autonomie endet im Gegenteil, nämlich in der noch größeren inneren Unfreiheit. Das Unbewusste kehrt bekanntlich wieder, es sucht immer neue Reinszenierungen in der Hoffnung, eines Tages einen günstigen Ausweg zu finden und die Sache endet doch meist im Desaster.

\section{Die Lebenslüge}

Psychologisch besonders interessant und von Kunstschaffenden immer wieder aufgegriffen, ist die Lebenslüge, die ebenfalls sehr stark durch innere Erlebniszustände motiviert ist. Sie ist psychologisch deshalb interessant, weil es sich um einen Grenzfall des Lügens handelt. Im Einzelfall ist nämlich nicht ganz präzise zu definieren, ob der Lügende sich tatsächlich bewusst ist, im Sinne einer bewussten, nachvollziehbaren Entscheidung, dass er sich selbst belügt. Ein interessantes Beispiel ist z.B. die sexuelle Orientierung eines Menschen, der einen gegengeschlechtlichen Menschen heiratet, um sich nicht

\footnotetext{
${ }^{9}$ Meist Eltern, Schule und Kirche.
} 
einzugestehen, dass er homosexuell ist. So gibt es viele Konstruktionen, in denen Menschen im Grunde lebenslänglich versuchen, sich etwas vorzumachen und es bleibt häufig ganz und gar offen, wie viel Bewusstsein und wie viel Entscheidungsfähigkeit dahinter steht. Die Lebenslüge hat fast immer tragische Seiten und es ist im psychologischen Sinne meist eine Verleugnung enthalten. Verleugnung wäre dann als Abwehrmechanismus zu verstehen, der per Definition nicht bewusst eingesetzt wird, der aber immer ganz nahe am Rande der Bewusstseinsfähigkeit ist. Insofern scheint es gerechtfertigt, die Lebenslüge in den vorliegenden Kontext (mit Vorbehalt) zu integrieren.

\section{Lügen zur Regulierung sozialer Beziehungen}

Hier spielt die Beziehungsstruktur zwischen Sender und Empfänger, also auch die Interaktion eine zentrale Rolle. $\mathrm{Zu}$ unterscheiden sind folgende Formen: Alltagslügen, Bequemlichkeitslügen, Notlügen und schließlich die Untreue in der Partnerschaft.

\section{Alltagslügen}

Man versteht darunter Lügen, die so alltäglich sind, dass sie schon eigentlich nicht mehr als Lüge zu bezeichnen sind, weil auch der Belogene im Grunde damit rechnet und implizit möglicherweise einverstanden ist mit dem Ritual. Aus empirischen Forschungen wissen wir einiges über die Häufigkeit, die geschlechtsspezifische Verteilung und die Bewertung des Verhaltens. ${ }^{10}$ Ganz einfache Beispiele sind etwa: Ein Mensch ruft an und man äußert Freude und Dankbarkeit über den Anruf, etwa nach dem Motto: „Ach wie schön, dass du anrufst!“ Zugleich kommt der Anruf allerdings ungelegen, man hat eigentlich keine Lust oder Zeit, ihn anzunehmen. Das Gleiche gilt, wenn man auf der Straße jemanden trifft und sagt: „Ach, wir müssten wieder einmal einen Kaffee trinken gehen“ oder „Du siehst ja blendend aus“ oder ähnliches. Dies sind Alltagsformulierungen, die implizit eine Lüge enthalten (können), die aber zivilisatorisch erlaubt und konventionell gefordert sind.

\section{Die Lüge aus Bequemlichkeit und zur Aufrechterhaltung einer Beziehung}

Ein gar nicht seltenes, sogar alltägliches Motiv der Lüge ist der Wunsch, sich Ungemach und Aufwand zu ersparen. Das Motiv ist, die Beziehung

\footnotetext{
${ }^{10}$ Sonja Ausserbauer, Isabell Beck, Katharina PhilipP, Silvia WARTEnsteiner, „Die Häufigkeit von Lügen im Alltag“, Semesterarbeit Universität Regensburg: http://www-app.uniregensburg.de/Fakultaeten/PPS/Psychologie/Lukesch/downloads/forschung/Berichte/ss03_3206 7 LuegenImAlltag.pdf.
} 
zwischen Sender und Empfänger unkompliziert und frei von Störungen zu gestalten (evtl. auch, um eine Geschäftsbeziehung nicht zu destabilisieren). In vielen Fällen vereinfacht eine kleine Lüge die Kommunikation, vermeidet Nachfragen, Einwände oder Zudringlichkeiten. Je nach den Erziehungsgrundsätzen, denen man selbst unterlag, lernt man, dass es in vielen Fällen sinnvoll ist, unter dem Radarsystem anderer Menschen zu segeln und hierfür alle Tricks der Darstellung und des Verschweigens anzuwenden. Dies kann im narzisstischen Sinne auch so weit gehen, dass man Erfolge und Fähigkeiten versteckt, um nicht von Anderen beneidet oder enteignet zu werden.

Hierzu gehört auch eine komplizierte und meist leidvolle Kunst des stützenden Lügens: die Fähigkeit nämlich, das narzisstische Gleichgewicht des Gegenübers zu erfassen und sich spielerisch darauf einzustellen und sozusagen die narzisstische Balance des Gegenübers so zu beeinflussen, dass möglichst große Stabilität herrscht (den andern stützen). Dies ist ein Manöver, das zwischen Kindern und Eltern, aber auch zwischen Ehepartnern sehr häufig zu finden ist. Dieses Verhalten gehört zur Lüge, weil man im Allgemeinen auch sehr genau weiß, dass man jetzt eine Manipulation vornimmt, den anderen lobt und beruhigt oder ihm schmeichelt oder ablenkt. (Bei Kindern ist in diesem Fall kaum von Lüge zu sprechen).

\section{Die Notlüge}

Die Notlüge ist vermutlich die einzige Lüge, die unbestritten moralisch hochstehend ist.

Sie ist immer damit verbunden, dass man durch die Lüge einem anderen Menschen etwas Gutes tut (mindestens tun möchte). So überlegt man sich etwa am Krankenbett eines Patienten, der auf der Intensivstation mit dem Leben kämpft, ob man ihm mitteilen möchte, dass ein guter Freund von ihm gerade gestorben ist. Wenn man entscheidet, dies nicht auszusprechen, so ist dies eine Lüge. Es handelt sich hier aber um eine Kontextualisierung, die zivilisatorisch akzeptabel erscheint und von der man davon ausgehen kann, dass der andere sie im Nachhinein verzeihen wird. Derartige Notlügen sind durchaus häufig im Alltag, sie genießen aber den Schutz der moralischen Begründbarkeit, die im Einzelfall natürlich auch immer höchst zweifelhaft und auch mit viel Leid verbunden sein kann.

\section{Lügen in der Partnerschaft}

Aus psychologischer Sicht dürften die im Rahmen von Partnerschaft auftretenden Lügen, v.a. die zur Kaschierung von Untreue besonders bedeutsam sein. Den deutschen Soziologen Beck und Beck-Gernsheim 
zufolge $^{11}$ hat die Partnerschaft in ihrer Bedeutung heute intrapsychisch den Rang der Religion. Insofern ist die Lüge in der Partnerschaft häufig auch eine der schwersten individuellen Belastungen und Krisen.

Lügen innerhalb der Partnerschaft haben viele Ebenen, aber vermutlich ist die sexuelle Untreue der zentrale Punkt. Hier gibt es unendlich viele Formen und Varianten, die zu diskutieren den Kontext dieses Beitrages sprengen würden. Jedenfalls ist die Lüge rings um die Untreue sehr verbreitet (30 bis $40 \%$ aller Menschen in fester Partnerschaft schlafen irgendwann mit anderen Partnern). Die unterliegenden psychologischen Motive sind vielfältig: Rache, Langeweile, narzisstische Balanceprobleme, Bindungsangst, Midlife-Krise, Triebdruck u.a.

\section{Weitere besondere Spielarten der Lüge}

Als Grenzfälle müssen folgende Lügenkonstruktionen erwähnt werden:

So spricht man etwa über den unverbesserlichen oder auch notorischen Lügner: hier liegt ein chronifiziertes, von der Person fast nicht beherrschbares zwanghaftes Verhalten vor. Man kann hier nicht von einer wirklichen Lüge sprechen, denn es sind zumeist schwere psychiatrische Erkrankungen, die hier hervortreten.

Weitere Beispiele illustrieren die Grenzsituationen:

Menschen, die sich selbst verletzen oder mit Substanzen krankheitsähnliche Zustände erzeugen (Münchhausen-Syndrom) und im Medizinsystem das eigene Zutun) verschweigen.

Gleiches gilt für Menschen, die dies nicht bei sich selbst, sondern bei ihren Kindern tun (Münchhausen bei Proxy-Syndrom). Hier ist der Schädigungsakt durchaus bewusst und muss mit Hilfe von Beweismitteln gesichert werden (versteckte Kameras). Die Kinder müssen geschützt werden und in manchen Ländern erfolgt die Bestrafung, weil eine Bewusstheit zu Recht angenommen wird.

Schließlich gibt es Menschen, die das Selbe tun, aber in einem zutiefst religiösen Kontext, die sich Stigmatisierungen ${ }^{12}$ zufügen und häufig in grenznahen mentalen Zuständen sind. In diesem Fällen handelt es sich zwar um prinzipiell bewusstseinsfähige Handlungen, aber häufig geschehen sie in einem Zustand tiefster Dissoziation oder aber im Rahmen von schweren psycho-pathologischen, das heißt psychiatrischen Störungen. Sie enthalten bewusstseinsfähiges Material, sind aber im individuellen Kontext doch von so massiven Wahrnehmungsstörungen begleitet, dass man sie im eigentlichen Sinne nicht mehr als Lüge bezeichnen kann. Sie sind daher auch nicht straffähig.

\footnotetext{
11 Ulrich BECK/ Elisabeth BECK-GERNSHEIM, Das ganz normale Chaos der Liebe, Frankfurt/M., Suhrkamp,1990.

${ }^{12}$ Gerd OVERBECK/ Ulrich NiEMANN, Stigmata, Geschichte und Psychosomatik eines religiösen Phänomens, Darmstadt, Wissenschaftliche Buchgesellschaft, 2012.
} 


\section{Tricks des Lügens und ihre Erfolgsaussichten}

Es werden nun verbreitete Lügentechniken charakterisiert und ihr jeweils unterschiedliches Risiko eingeschätzt.

\section{Weglassen und Ablenken}

Das Weglassen als Versuch, bestimmte Sachverhalte nicht oder nur teilweise ans Licht kommen zu lassen, ist psychoökonomisch sicher die „günstigste“ Variante der Lüge. ${ }^{13}$ Das Ablenken von einem Thema, das Einbringen von Alternativthemen oder der Versuch, durch Aktionen und gezielte Aufmerksamkeits(ab)lenkung vom Sachverhalt wegzukommen, ist ebenfalls psychisch nicht allzu aufwändig und für Geübte unproblematisch.

Man muss bei diesen Techniken nichts erfinden, muss keine (unwahren) „Geschichten erzählen“, kann sich nicht in (nachweisliche) Widersprüche verwickeln und es bleibt immer eine Korrekturlücke, indem man - wenn es eng wird - mit kleinen Portionen Nachreichungen vornimmt. Das Weglassen erfordert auch wenig Aufwand hinsichtlich der Modulation der Körpersprache und der Emotionsunterdrückung, wenngleich auch hier Signale gesendet werden können, die geübten Beobachtern als Hinweise gelten.

\section{Über- oder Untertreiben}

Das Über- oder Untertreiben ist psychoökonomisch sehr viel aufwändiger. Hier müssen Fakten und Gefühle entweder vorgespielt oder aber Gefühle unterdrückt werden. Beides ist fehlerträchtig, weil noch nicht einmal gute Schauspieler einen vollständigen willentlichen Zugriff auf die komplexen muskulären Muster haben. Damit ist das Risiko der Aufdeckung groß. Allerdings bleiben auch hier Auswege und (halbwegs) ehrenvolle Abgänge, denn es ist schließlich nicht möglich, einen schlüssigen Nachweis des willentlichen Lügens zu führen, da das Unter- oder Übertreiben eher als emotionale Fehler klassifiziert werden, die moralisch nicht so verwerflich sind wie die ,glatte“ Lüge.

\section{Erfinden und Verfälschen}

Erfinden und Verfälschen gehören $\mathrm{zu}$ den risikoreichsten Täuschungsmanövern. Wenn man nicht wirklich abgebrüht und geübt ist (wie etwa Politiker es aufgrund ihres langen Weges an die Macht), dann erfordert das Erfinden sehr viel psychischen Aufwand. Die Geschichte muss in sich

\footnotetext{
${ }^{13}$ Die halbe Wahrheit sagen oder verschweigen (weglassen) kann als kleines Missgeschick, als ,versehentliche“ Verdrehung oder Verharmlosung attribuiert werden.
} 
konsistent und widerspruchsfrei sein, sie muss zu den feststellbaren Fakten passen, sie muss Motive und Bedenken berücksichtigen und man muss sich alle erzählten Details sehr genau merken, um nicht sofort verräterische Ungereimtheiten zu erzeugen. All das erfordert größten Aufwand, höchste Konzentration und ist überdies gefährlich, weil kleinste Details zur Entlarvung beitragen können. Die Aufdeckungsgefahr bleibt über Jahre und Jahrzehnte groß, weil jedes Detail die Geschichte in Lügenverdacht bringen kann. Eine sehr verbreitete Technik ist: Im Alltag Routineabläufe über lange Zeit zu etablieren, bis sie nicht mehr hinterfragt werden. Dann können diese Routinen als Platzhalter (z.B. für außereheliche Beziehungen) verwendet werden.

\section{Vergessensbehauptung}

Eine sehr beliebte Technik ist die Behauptung, etwas vergessen zu haben. Diese Technik ist in manchen Bereichen durchaus erfolgversprechend und bei Politikern sehr verbreitet. Man kann hier zum Beispiel immer behaupten, man habe etwas nicht gewusst und kann sogar dann noch einen Rettungsversuch wagen, wenn Beweise für das Gegenteil vorliegen (z.B. eine Unterschrift unter eine Aktennotiz u.ä.). Man hat dann zwar die Verantwortung zu übernehmen, kann aber noch eine Ehrenrettung versuchen, weil man völlig überlastet und überfordert war und so vieles täglich zu unterschreiben hatte, dass es zu solchen Fehlhandlungen „leicht" kommen konnte.

Die sehr kurze Darstellung der Motivstränge, der Mechanismen und Kontextualisierungen des Lügens zeigt, dass es eine in jedem Lebensbereich auftretende Verhaltensweise ist, die daher auch eine außerordentliche Vielfalt hat. Im Grunde ist die Lüge ein Joker des Sozialverhaltens, der je spezifisch und individuell gefärbt in jeder Gefühlslage, in allen erdenklichen Konfliktkonstellationen und allen sozialen Kontexten zur Anwendung kommen kann. Möglicherweise ist dies der Grund dafür, dass es kein psychologisches Lehrbuch der Lüge gibt.

\section{Der Lügendetektor}

Die ersten Überlegungen zu einem sogenannten Lügendetektor stammen von C. G. Jung ${ }^{14}$ und M. Wertheimer ${ }^{15}$. Eine Realisierung gelang durch den Bau des ersten Polygraphen ca. 1935.

\footnotetext{
${ }^{14}$ Es ist eindrucksvoll, wie differenziert und methodisch ausgeklügelt C. G.Jung in den ersten Jahren seines Schaffens psychophysiologische Forschung in Angriff nahm und in englischer Sprache publizierte. Der zweite Band seiner 23-bändigen Gesamtausgabe dokumentiert dies eindrucksvoll. Die Arbeiten wurden 1907 in angesehenen internationalen Zeitschriften publiziert. Danach hat er seinen Arbeitsschwerpunkt vollständig verlagert. Carl Gustav JUNG, Experimentelle Untersuchungen, Gesammelte Werke, Bd. 2, Solothurn, Walter Verlag, 1995 .
} 
Bis heute ist der Korpus an wissenschaftlicher Literatur zu diesem Thema außerordentlich groß und nahezu unüberschaubar. ${ }^{16}$ Die Wissenschaften, die sich damit beschäftigen, sind zahlreich, vor allem sind das die Psychophysiologie, die Juristerei und die Militärwissenschaft. Alle haben völlig unterschiedliche Interessen und Fragestellungen an den Polygraphen.

\section{Was ist die Logik des Polygraphen?}

Die psychophysiologische Forschungsfrage ist seit vielen Jahrzehnten folgende: Da am Verhalten des Lügens komplexe emotionale und kognitive Prozesse beteiligt sind und körperliche Stressreaktionen des autonomen Nervensystems (die willentlich nicht beeinflusst werden können) messbar sind, müsste es möglich sein, anhand dieser Parameter Aussagen als wahr oder erlogen zu differenzieren.

Von dieser Voraussetzung ausgehend, hat man eine ganze Reihe von psychophysiologischen Messgrößen wissenschaftlich getestet: Hautwiderstand, Puls, Herzfrequenzvariabilität, Hauttemperatur, Muskeltonus, Blutdruck und Atmung. Heute werden zunehmend auch Messungen mittels MRT herangezogen. ${ }^{17}$ Die Forschungsmethoden und die Verhörtechniken sind sehr ausgeklügelt und aufwändig.

Das Ergebnis all' dieser Studien ist bisher sehr widersprüchlich: Es gibt nur wenige absolut spezifische autonome Reaktionsmuster, die mit immer denselben Emotionen sicher verknüpft werden können. Alles in allem sind die autonomen Funktionen eher generalisiert-gleichförmig im Sinne der Aktivierungsachse Flucht versus Kampf bzw. Sympathikus versus Parasympathikus. Da das Verhaltensmuster Lügen in erster Linie eine körperliche Stressreaktion auslöst, ist die Interpretation der Messungen immer unsicher.

Beispiel für eine gängige Argumentationslogik: Man geht davon aus, dass ein Mensch, der die Wahrheit sagt, eine geringere Gehirnanstrengung zu leisten hat als ein Mensch, der lügt. Das Lügen erfordert mehr Energie, mehr neuronale Prozesse und die Beteiligung von mehr Gehirnregionen und dauert daher länger. Diese Hypothese führt zu ausgeklügelten gezielten Assoziationstests oder Materialpräsentationstests. Die in Millisekunden messbaren Reaktionszeiten auf standardisierte Aufgabenstellungen werden als Indikatoren verwendet: Je länger die Reaktionszeit im Vergleich zu vorliegenden Erfahrungswerten, desto wahrscheinlicher ist eine Lüge.

\footnotetext{
${ }^{15}$ Wertheimer hatte schon in seiner Dissertation (erschienen 1905) empirisch und sehr intelligent über objektive Tatbestandsdiagnostik geforscht.

${ }^{16}$ Umfassend informiert die medizinische Datenbank Pubmed.

17 vgl. M. RUCHSOW/ L. HERMKE/ M. KOBER, „MRT als Lügendetektor und Gedankenleser? Kritische Bestandsaufnahme und Reflexion“, Der Nervenarzt, 81, 2010, S. 1085-1091.
} 
Der wissenschaftliche Haupteinwand lautet: Zufällige Versuchspersonen im Labor ${ }^{18}$ haben keinerlei Interesse und stehen nicht unter Druck. Der große Korpus an wissenschaftlicher Literatur ist daher zunächst einmal überhaupt nicht auf die Realität juristischer Tatbestände oder Verhörmethoden der Armeen übertragbar. Menschen, die einer Untersuchung mittels Lügendetektor zustimmen bzw. dazu gezwungen wurden, können ihre Motive für sich behalten und absichtlich unkooperatives Verhalten an den Tag legen.

Es gibt ein Faktum, das die dahingehenden Einwände präzise auf den Punkt bringt: Die amerikanische und auch andere Armeen, trainieren ihre Soldaten systematisch hinsichtlich spezifischer Verhörmethoden. Sie trainieren, damit ihre Soldaten, wenn sie in Gefangenschaft geraten, durch die mittels Lügendetektor gemessenen autonomen Reaktionen nicht Geheimnisse an den Gegner verraten. Das Training ist im Grunde sehr einfach: Man kann nicht trainieren, dass es beim Lügen keine Ausschläge in den verschiedenen Parametern gibt, man kann aber leicht trainieren, dass es bei allen Fragen heftige Ausschläge gibt, indem man systematisch und bewusst das autonome Nervensystem aktiviert. Dies kann durch verschiedene Techniken jeder Mensch erlernen. Damit haben alle diese Verhörmethoden einen systematischen Fehler in die Richtung der Hochaktivierung, was dazu führt, dass der Polygraph auch bei unbedeutenden Fragen (und daher auch bei der Artikulation der Wahrheit) heftig ausschlägt.

\section{Was ist die Zukunft dieses Verfahrens?}

Im Moment zeichnet sich ab, dass mit dem wissenschaftlich-technischen Fortschritt immer weitere autonome Funktionen, vor allem auch neurophysiologische Funktionen, in die Messung einbezogen werden. Das MRT ist hierfür eine geeignete Forschungsmethodik. Allerdings ist das MRT für die Frage der Wahrheitsfindung ebenfalls durch den genannten systematischen Messfehler behaftet: Er setzt eine hohe Kooperationsbereitschaft voraus, weil man in dem Gerät sehr still liegen und kooperationsbereit sein muss. Und es ist wohl kaum vorstellbar, dass Politiker und Banker ins MRT geschoben werden, um Wahrheiten zu erfahren.

\section{Körpersprache und Lügen}

Der Forscher Paul Ekman wird oft als der beste Lügendetektor der Welt bezeichnet. Was bedeutet dies?

Es gibt einen großen Forschungszweig, der sich systematisch und mit hohem apparativem Aufwand mit der Dechiffrierung der Körpersprache beschäftigt (u.a. R. Krause in Deutschland). ${ }^{19}$ Ekman hat ein komplexes

\footnotetext{
${ }^{18}$ Das sind in der psychologischen Forschung zumeist Psychologiestudenten.

${ }^{19}$ vgl. http://www.pukzh.ch/default/assets/File/Handout_Prof._R._Krause.pdf
} 
System zu Codierung der Gesichtsmuskulatur entwickelt: Das FACS (Facial Action Coding System). Moderne Softwaresysteme der Gesichtserkennung sind in der Lage, die komplexen Muster der menschlichen Ausdruckskonstellationen zu kartografieren.

Ekman ${ }^{20}$ hat durch extrem feine Untersuchungsmethoden Reaktionen von Muskelgruppen erforscht, die dem normalen Menschen entgehen. Er hat in Videoanalysen herausgefunden, dass es Reaktionsmuster in der Gesichtsmuskulatur gibt, die nur im Bruchteil einer Sekunde auftreten (und daher nicht bewusstseinsfähig sind), die aber nach seinen Erfahrungen ein deutlicher Hinweis darauf sind, ob es sich um echte oder simulierte emotionale Gesichtsausdrücke handelt.

Am Beginn seiner Forschung hatte er eine Videoaufnahme von einer psychiatrischen Patientin, die eindeutig lügt, was man aber erst später erkennen konnte (sie verübte kurz nach dem Gespräch Selbstmord, hatte aber im Gespräch glaubhaft versichert, sich von solchen Gedanken distanziert zu haben). Hunderte Male hat er mit Kollegen das Band angesehen und nichts gefunden, was auf das fatale Ende hindeutete. Erst als er in Zeitlupe Bild für Bild ansah, hat er die „verräterischen“ Mikroprozesse entdeckt. Für Sekundenbruchteile waren mimische Muster zu erkennen, die zu den übrigen kontrastierten und den Suizid ankündigten. Wir müssen davon ausgehen, dass solche Reaktionsmuster von unserem Gehirn aufgenommen und verarbeitet werden, dass sie sogar prozessiert werden und handlungsleitende Funktionen erfüllen, dass sie aber dennoch nicht dem Bewusstsein zugänglich sind. ${ }^{21}$

Diese Mikroprozesse haben eine hohe Bedeutung und werden sich in Zukunft sicher der Forschung noch weiter öffnen. Man ist in der Lage, durch Filmaufnahmen und Computerauswertungen diese Vorgänge genauer zu erfassen und es ist sehr wahrscheinlich, dass die Muster von gleichzeitig aktiven Muskelgruppen bei Emotionen wie etwa Angst, Freude, Lachen etc. so spezifisch sind, dass man mit dieser Technik durch eine nachträgliche Analyse sehr viel erfahren kann.

In der Wissenschaft ist man im Moment dabei, diese verschiedenen Reaktionsmuster zu kategorisieren und einer systematischen Auswertung zuzuführen.

Die Grundthese hinsichtlich der Lügendetektion ist also: Ein Mensch, der eine echte aufrichtige Emotion zeigt, hat ein anderes Muster des muskulären Zusammenspiels als ein Mensch, der lügt bzw. eine Emotion nur vorspielt. Vor allem die Gesichtsmuskulatur, daneben aber auch die Bewegungen der

\footnotetext{
${ }^{20}$ Paul EKMAN, Ich weiß, dass du lügst. Was Gesichter verraten, Reinbek, Rowohlt, 2011, sowie "What the Face Reveals: Basic and Applied Studies of Spontaneous Expression Using the Facial Action Coding System (FACS)", Series in Affective Science, Oxford, University Press, 2005.

${ }^{21}$ Faszinierend ist, dass man an in Gefangenheit lebenden Schimpansen zeigen konnte, dass sie in Lernexperimenten auf Reizdarbietungen korrekt reagieren konnten, die von der Präsentationszeit weit unter dem liegen, was Menschen erkennen können.
} 
Hände und der Arme werden einbezogen. ${ }^{22}$ Das Gesicht hat 24 Muskeln. Einige Halsmuskeln modulieren zusätzlich den Gesamtausdruck und werden vom Empfänger dechiffriert. Die Kombinationen der Muskelbewegungen in Mimik und Gestik sind außerordentlich komplex: die Kombination der 24 Muskelgruppen ergibt eine Variationsbreite von ca. 10.000 Kombinationen, da beim Ausdruck jeder Emotion immer mehrere Muskeln gleichzeitig beteiligt sind. Damit ergibt sich am Ende eine äußerst komplexe, aber eindeutige Interpretation. Nach heutigem Wissen geht man hinsichtlich der emotionalen körpersprachlichen Ausdrucksweisen von einer Universalität aus, das heißt es wird vermutet, dass zentrale, lebenswichtige Ausdrucksformen bei allen Menschen gleich sind.

Nun scheint es wenige Menschen zu geben, die die außerordentliche Begabung haben, mimische Zusammenhänge (Mikromimik) in Sekundenbruchteilen sozusagen intuitiv, also ohne besondere Anstrengung, zu erfassen und zu interpretieren (aber auch sie können sie nicht bewusstseinsfähig machen und erläutern). Diese Menschen können durch hohe Aufmerksamkeit und zusätzliches Training die Reaktionsmuster anderer schnell und genau erfassen und sie werden beim Militär und im Grenzzoll gerne eingesetzt. Es ist tatsächlich verblüffend ihnen zuzuschauen. Sie können meist nicht beschreiben, aufgrund welcher Indizien sie zu einer Einschätzung kamen. Es scheinen hochkomplexe Mustererkennungen zu sein.

Intuitiv können vermutlich fast alle Menschen, wenn sie psychisch halbwegs stabil und gesund sind, sehr viel mehr erfassen, als ihnen bewusst wird. Insgesamt gehen wir davon aus, dass die Wahrnehmung dem Menschen nur zu etwa $10 \%$ bewusst zur Verfügung steht, das heißt $90 \%$ des Wahrgenommen wird zwar gespeichert und auch analysiert, aber nicht der bewussten Bearbeitung zugeführt.

Die Schlussfolgerung mag ernüchternd sein: Es wird in den nächsten 15 Jahren Fortschritte in der Wissenschaft geben, die aber, nach allem was wir über unsere Gesellschaft wissen, keineswegs relevante Veränderungen bewirken werden hinsichtlich der „großen“ Lügner unserer Welt, die eingangs des Kapitels genannt wurden (in Politik, Banken, Religionsgemeinschaften und Werbeindustrie).

Aus diesen Forschungen ergibt sich eine Empfehlung an alle Lügner: Emotionen, die man nicht hat, sind schwer vorzuspielen und die Unterdrückung von heftigen Emotionen ist ebenfalls außerordentlich fehlerträchtig. Das bedeutet: Man kann nur eine Maßnahme trainieren, nämlich soweit wie irgend möglich die Gesichtsmuskeln still zu halten, außerdem unbedingt die Hände bewegungslos nach unten hängen zu lassen und dann mit gleichförmiger Stimme zu sprechen.

\footnotetext{
${ }^{22}$ Einen Finger ins Gesicht führen gilt als sehr verräterisch.
} 